\title{
El deterioro de las condiciones laborales de los corresponsales de radio
}

Deteriorating working conditions for radio correspondents

Juan Tato Suárez, Universidad Carlos III de Madrid - jtato@hum.uc3m.es

\section{Resumen}

La situación laboral de los corresponsales de radio en el extranjero ha venido deteriorándose de forma progresiva a lo largo de los últimos años. El empeoramiento de sus condiciones de trabajo obedece a una doble causalidad. Por un lado, es consecuencia de la crisis económica que sufren las empresas radiofónicas en un entorno mediático cada vez más fragmentado y competitivo. Por otro, de los desarrollos tecnológicos en los campos de la informática y las telecomunicaciones, que han multiplicado la capacidad de recibir y transmitir información al instante desde cualquier punto del planeta y abaratado el coste de las conexiones desde el extranjero, incrementando, como consecuencia, la carga de trabajo de los corresponsales. Esta investigación intenta determinar cómo se han modificado las condiciones de trabajo de los corresponsales de radio en el extranjero y cómo los cambios experimentados han afectado a sus retribuciones, a la rutina de su jornada y a su estabilidad laboral.

\section{Palabras clave}

Corresponsales extranjeros; radio; información internacional; precariedad laboral.

Abstract

The labour situation of radio foreign correspondents has steadily deteriorated over these past years. The worsening of their working conditions has a double cause. On the one hand, it is a consequence of the economic crisis suffered by many radio companies in an increasingly fragmented and competitive media scene. On the other hand, technological development in the fields of computing and telecommunications has multiplied the capacity to broadcast information instantly from every corner of the planet and lowered the price of connections from abroad. As a consequence the workload of foreign correspondents has increased substantially. This investigation wants to determine how the working conditions of radio foreign correspondents have been modified and in what way have those changes/modifications affected their retribution, their daily routine and their job stability.

\section{Keywords}

Foreign correspondents; radio; international news reporting; poor working conditions.

Sumario

1. Introducción. 2. Metodología. 3. Resultados. 4. Reflexiones de los corresponsales de radio sobre su futuro laboral. 5. Conclusiones. 6. Bibliografía 


\section{Introducción}

El objetivo de esta investigación es estudiar cómo la crisis económica que sufren las empresas radiofónicas está afectando a la situación laboral y a las condiciones de trabajo de los corresponsales de radio en el extranjero.

El advenimiento de la era digital ha cambiado el ecosistema de los medios de comunicación, facilitando el surgimiento de nuevas plataformas y maneras alternativas de elaborar y distribuir información. Durante las últimas dos décadas los medios tradicionales han sobrevivido en un estado permanente de crisis, intentando adaptarse al nuevo entorno económico para garantizar su viabilidad. La mayor parte de ellos -radios incluidas- han visto deteriorada su situación financiera y, como consecuencia, han tenido que recortar la retribución de su masa de asalariados 0 , en los casos más extremos, reducir su plantilla de periodistas.

Son múltiples los autores que dan cuenta de la reducción que en los últimos tiempos ha habido en el número de corresponsalías operadas por los medios de comunicación occidentales en general y españoles en particular. (Sambrook, 2010; Rábago, 2012; Molina, 2012; Enríquez, 2012; Sahagún, 2013; Cohen, 2015, Terzis, 2015). Al menos, tal y como las conocíamos en el sentido clásico, con el establecimiento de una oficina permanente en el extranjero, dedicación exclusiva al medio y una situación laboral estable. Durante más cien años la principal forma de conocer lo que ocurría en el resto del mundo era a través de la información provista por los corresponsales extranjeros pero ahora «estamos entrando en una nueva era en la que los corresponsales ya no son una pieza central para saber qué pasa en el mundo» (Sambrook, 2010: 1).

En España, a lo largo de los últimos años, las principales cadenas generalistas de radio han reducido el presupuesto destinado a la cobertura de información internacional y han recortado sus plantillas de corresponsales fijos (Sahagún, 2013: 17).

En la actualidad, Radio Nacional de España, que se financia a través de los presupuestos generales del estado y que no opera con criterios de rentabilidad empresarial, es la única de las grandes cadenas de radio de España que mantiene una red estable de corresponsales. Periodistas profesionales, miembros de la plantilla de la empresa, a los que se traslada al extranjero para ocupar una determinada corresponsalía. En los últimos años RNE ha cerrado las corresponsalías de Moscú (2011), Pekín (2013) y San José de Costa Rica (2014), pero ha suplido la ausencia de un corresponsal propio en esas ciudades haciendo uso de los corresponsales que comparte conjuntamente con TVE.

Paralelamente en las radios privadas el uso de corresponsales freelance se ha generalizado de forma progresiva en detrimento de los corresponsales fijos (Molina, 2012: 8).

Las radios españolas atraviesan dificultades económicas considerables. En el caso de la radio pública, los ingresos de RNE se han visto mermados al haberse reducido de forma sustancial, con motivo de la crisis económica, la aportación anual que recibe del estado a cuenta de los presupuestos generales.

En el caso de las empresas radiofónicas privadas sus problemas económicos nacen en buena medida de la disminución de la inversión publicitaria en la radio que ha caído cerca del 30\% desde el año 2010 (Asociación de la Prensa de Madrid, 2015: 71). El desplome de los ingresos tiene un impacto directo en la calidad del empleo de los periodistas radiofónicos. La Asociación de la Prensa de Madrid (APM) denuncia en su informe de 2015 que en términos generales se ha producido «un aumento de la precariedad en las condiciones de trabajo de periodistas y comunicadores, quienes, por su parte, vuelven a señalar este problema como la principal dificultad profesional» (Asociación de la Prensa de Madrid, 2015: 39).

\section{Metodología}

Para llevar a cabo esta investigación se ha optado por estudiar la situación laboral de los corresponsales en el extranjero de las cuatro cadenas de radio generalista más escuchadas de España: Cadena SER, Cadena COPE, Onda Cero y Radio Nacional de España. La muestra seleccionada se circunscribe a las cuatro grandes cadenas de radio convencional que según el Estudio General de Medios ( $2^{\mathrm{a}}$ Ola de 2016) representan, de forma agregada, el 75'9\% del share de la radio generalista en España.

Siguiendo los ejemplos de investigaciones similares sobre corresponsales en el extranjero (Sahagún, 1986; Tulloch, 1998), se ha seleccionado la encuesta (Sierra Bravo, 1994; Cea d'Ancona, 1998; Vinuesa, 2005) como técnica de investigación para tratar de describir de la forma más fidedigna posible las condiciones de trabajo de los corresponsales de radio en el extranjero.

Las cuatro grandes cadenas de radio cuentan conjuntamente con 64 corresponsales en el extranjero desplegados en 24 países. A todos ellos se les hizo llegar un cuestionario distribuido a través de correo electrónico con preguntas acerca de sus retribuciones, estructura salarial, dedicación exclusiva o parcial a la radio, etc. Se recibieron en total 61 respuestas de 64 posibles lo que equivale a un porcentaje del $95,31 \%$. El trabajo de campo se realizó durante 2015 , si bien entrado ya 2016 se admitieron los cuestionarios de algunos corresponsales que se habían demorado en su entrega.

\section{Resultados}

De acuerdo con los datos obtenidos en el marco de esta investigación, la mayoría de los corresponsales de radio (53\%) son freelance, mientras que solo el $47 \%$ son fijos. Si se descuentan los corresponsales de RNE, que son todos fijos, se concluye que 
en las emisoras privadas de radio el $69 \%$ de los corresponsales son freelance, mientras que solo el $31 \%$ son fijos.

Cuánto más jóvenes son los corresponsales, más inestable es su situación laboral. Los seis corresponsales menores de 30 años son todos freelance. De la misma manera, de los 16 corresponsales que tienen más de 50 años, 14 son corresponsales fijos y solo dos son freelance.

Si atendemos a la estructura salarial de los corresponsales de radio constatamos que el $50 \%$ de ellos percibe un salario fijo, un $37 \%$ cobra por pieza, y un 10\% tiene un salario compuesto de una parte fija y de una variable. El $3 \%$ restante ha preferido no responder a la cuestión.

\title{
Figura 1. Estructura salarial de los corresponsales
}

\author{
¿Tiene un salario fijo, cobra usted por pieza, o tiene un salario compuesto de una parte \\ fija más una variable? \\ 60 de 61 personas han respondido esta pregunta
}

\begin{tabular}{|l|l|l|}
\hline 1 & Salario fijo & $30 / 50 \%$ \\
\hline 2 & Cobro por pieza & $22 / 37 \%$ \\
\hline 3 & Salario fijo + variable & $6 / 10 \%$ \\
\hline 4 & Prefiero no responder & $2 / 3 \%$ \\
\hline
\end{tabular}

Fuente: Elaboración propia

El resultado está distorsionado por los datos de los catorce corresponsales de RTVE, que perciben un salario fijo. Si nos centramos únicamente en los corresponsales de las radios privadas obtenemos una radiografía bien diferente. El $47 \%$ cobra por pieza, el $34 \%$ tiene un salario fijo, y el $13 \%$ un salario integrado por una parte fija y un complemento variable. El $4 \%$ ha preferido no responder.

En relación con su retribución, el $37 \%$ de los corresponsales en el extranjero encuestados asegura que recibe de la radio un salario bruto anual inferior a los $15.000 €$; el $19 \%$ asegura cobrar más de $30.000 €$ y el $10 \%$ gana entre $15.000 €$ y $30.000 €$. El $34 \%$ de los corresponsales encuestados ha preferido no contestar a la pregunta.

\section{Figura 2. Salario de los corresponsales}

\section{Salario que percibe de la radio (bruto anual)}

59 de 61 personas han respondido esta pregunta

\begin{tabular}{|l|lr|}
\hline 1 & menos de $15.000 €$ & $22 / 37 \%$ \\
\hline 2 & Prefiero no contestar & $20 / 34 \%$ \\
\hline 3 & más de $30.000 €$ & $11 / 19 \%$ \\
\hline 4 & entre $15.000 €$ y $30.000 €$ & $6 / 10 \%$ \\
\hline
\end{tabular}

Fuente: Elaboración propia

En las cadenas de radio privadas el $71 \%$ de los corresponsales recibe una retribución bruta por parte de la radio inferior a los $15.000 €$ anuales; el $19 \%$ cobra entre $15.000 €$ y $30.000 €$; y solo un $10 \%$ gana más de $30.000 €$. Esto significa que más de dos tercios de los corresponsales de las emisoras de radio privadas de España reciben un salario bruto anual que está por debajo del sueldo medio de España y también del llamado sueldo frecuente, que según el Instituto Nacional de Estadística se eleva hasta 
los $15.500 € 1$.

El $60 \%$ de los corresponsales de las radios privadas o bien cobran a la pieza o bien tienen una estructura salarial que combina un sueldo fijo y uno variable. La mayor de ellos $(77 \%)$ asegura que su empresa radiofónica les paga la crónica de radio con un importe que se sitúa entre los $30 €$ y los $40 €$. El $7 \%$ asegura recibir menos de $30 €$ por cada pieza enviada. El $17 \%$ ha preferido no responder.

Figura 3. El precio de las crónicas de radio

\section{¿A cuánto paga la radio para la que trabaja su crónica de corresponsal? \\ 30 de 61 personas han respondido esta pregunta}

\begin{tabular}{|l|l|l|}
\hline 1 & entre $30 €$ y $40 €$ & $23 / 77 \%$ \\
\hline 2 & Prefiero no contestar & $5 / 17 \%$ \\
\hline 3 & menos de $30 €$ & $2 / 7 \%$ \\
\hline 4 & entre $40 €$ y $50 €$ & $0 / 0 \%$ \\
\hline 5 & más de $50 €$ & $0 / 0 \%$ \\
\hline
\end{tabular}

Fuente: Elaboración propia

Los corresponsales que no disponen de un salario fijo tienen en general unos ingresos relativamente bajos que son además irregulares. En el ejercicio del trabajo de corresponsal se alternan cíclicamente periodos de alta actividad, coincidentes con la ocurrencia de acontecimientos de alcance, con otras etapas de menos intensidad informativa. Es justamente en estas últimas en las que los ingresos de los corresponsales que cobran por pieza se resienten de forma notable. Los corresponsales que tienen esta estructura salarial tienen más dificultades para planificar económicamente su futuro.

Muchos corresponsales en el extranjero consideran que su situación laboral es manifiestamente mejorable. Para muchos el dinero que perciben por el desempeño de su profesión es insuficiente y esto se traduce en que no están especialmente satisfechos con su salario.

Figura 4. Satisfacción de los corresponsales con su salario

Valore del 1 al 10 su grado de satisfacción con su salario como corresponsal de radio 55 de 61 personas han respondido esta pregunta

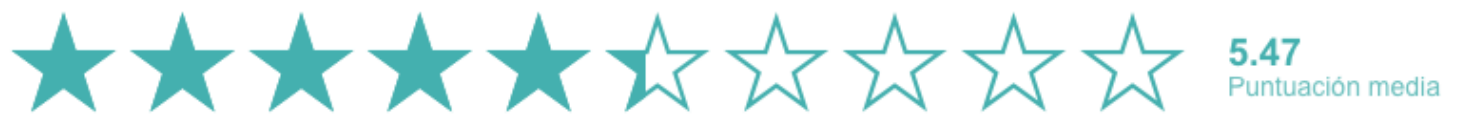

Fuente: Elaboración propia

A lo largo de los años la figura del corresponsal se ha ido transformando y a medida que lo ha hecho se ha ido también precarizando. Los bajos sueldos que reciben algunos corresponsales por parte de las emisoras de radio que contratan sus servicios les obligan a buscar fuentes de ingresos alternativas. De hecho, para el $53 \%$ de los corresponsales encuestados la radio no es su principal fuente de ingresos. Si desagregamos los datos, se observa que en las cadenas privadas el porcentaje de corresponsales para los que la radio no es su principal fuente de ingresos se eleva al $68 \%$. El $69 \%$ de los corresponsales en el

\footnotetext{
${ }^{1}$ Según el INE «la ganancia media anual por trabajador fue de 22.697,86 euros en 2013 (...). Una característica de las funciones de distribución salarial es que figuran muchos más trabajadores en los valores bajos que en los sueldos más elevados. Este hecho da lugar a que el salario medio sea superior tanto al salario mediano, como al más frecuente. Así, el salario mediano (el que divide al número de trabajadores en dos partes iguales, los que tienen un salario superior y los que tienen un salario inferior) presentó un valor de 19.029,66 euros en 2013. El sueldo más frecuente se situó en torno a 15.500 euros».
} 
extranjero se ven en la obligación de informar paralelamente, además de para la radio, para otros medios de comunicación.

Figura 5. El pluriempleo de los corresponsales

\author{
En la actualidad, además de la radio, ¿trabaja usted como corresponsal para otros \\ medios de comunicación? \\ 59 de 61 personas han respondido esta pregunta
}

\begin{tabular}{|l|l|r|}
\hline 1 & Si & $41 / \mathbf{6 9} \%$ \\
\hline 2 & No & $18 / 31 \%$ \\
\hline
\end{tabular}

Fuente: Elaboración propia

Los corresponsales del siglo XXI son periodistas versátiles, con una capacidad creciente para trabajar en múltiples plataformas y con un buen dominio de las tecnologías. La mayoría de los corresponsales están facultados para cubrir información para la radio, la prensa, la televisión o para publicaciones electrónicas y han demostrado sobrada capacidad para asumir todas las facetas de la producción informativa: la administrativa, la puramente periodística o la técnica.

Los corresponsales que, además de para la radio, trabajan para otros medios, suelen hacerlo en la prensa escrita (61\%), la televisión $(54 \%)$, las publicaciones digitales $(27 \%)$ y las agencias de noticias $(10 \%)$. Hay varios corresponsales que trabajan incluso para tres o cuatro plataformas diferentes.

Otro aspecto digno de ser destacado es que muchos corresponsales de radio han desarrollado aptitudes y capacidades que les permiten trabajar para medios de comunicación de otros países y por lo tanto competir en el mercado laboral internacional. El $27 \%$ de los corresponsales de las radios españolas que trabaja para otro medio lo hace para un medio de comunicación internacional.

De los resultados del cuestionario se desprende que al menos 38 corresponsales tienen como fuente de ingresos paralela un medio de comunicación distinto a la radio. Esto se traduce en que como mínimo un 59,3\% de los corresponsales de radio depende para su supervivencia de medios alternativos a las radio. En la mayoría de los casos el sueldo que los corresponsales perciben de la radio es en realidad un complemento salarial.

Los ingresos de algunos corresponsales de radio son a veces tan exiguos que en casos puntuales se ven, no ya en la obligación de trabajar para otros medios de comunicación, sino de hacerlo para empresas o negocios que nada tienen que ver con el mundo del periodismo. Seis de los 64 corresponsales que trabajan para las distintas emisoras de radio españolas realizan algún tipo de trabajo remunerado que no guarda relación con el periodismo.

A día de hoy son mayoría los corresponsales de radio que accedieron al puesto después de haberse ofrecido personalmente al medio. En muchos casos estos corresponsales no mantienen una relación laboral estable con la emisora de radio a la que prestan sus servicios, facturan como autónomos y se responsabilizan individualmente del pago de sus cotizaciones a la seguridad social en el país en el que residen. Poco o poco el modelo hegemónico en la segunda mitad del siglo XX, por el cual las empresas desplazaban a su personal al exterior, va desapareciendo. Antaño era el medio el que se solía hacerse cargo del pago de la vivienda del corresponsal en el extranjero. En el ámbito de las radios españolas solo RTVE -gracias a su particular régimen de financiación- sigue abonando a los corresponsales una ayuda económica que les permite sufragar parte del importe del alquiler de la casa-corresponsalía en la que trabajan.

De la misma manera que en tiempos pretéritos las empresas radiofónicas sufragaban el material y el equipo técnico necesario para que los corresponsales desempeñaran su trabajo, en la actualidad la mayor parte de los corresponsales de radio $(54 \%)$ son propietarios de su equipo técnico. Este fenómeno se apoya también en el progresivo abaratamiento de los medios tecnológicos e informáticos, que permiten al corresponsal de radio grabar y montar sus crónicas o reportajes y hacer conexiones fiables y seguras con buena calidad de sonido desde los puntos remotos en los que se encuentran.

Con carácter general el corresponsal de radio es la única persona que en representación de su medio se encarga de cubrir el país o la región que tiene encomendadas. Los medios que los emplean les suelen exigir disponibilidad absoluta. Las noticias pueden producirse a cualquier hora, cualquier día, y el corresponsal debe, por lo tanto, estar operativo las 24 horas durante los siete días de la semana para hacer frente a cualquier contingencia que pueda sobrevenir.

La información fluye de forma continua e incluso cuando la noticia se ha producido el corresponsal debe hacer un seguimiento de la misma y actualizarla de forma constante. No son pocas las ocasiones en las que en un mismo día se solapan varios acontecimientos relevantes obligando al corresponsal a realizar de forma simultánea coberturas múltiples. 
Las jornadas laborales de los corresponsales de radio suelen ser maratonianas. Cuando se les pregunta cuántas horas trabajan de media al día el $55 \%$ de los corresponsales responde que trabaja entre diez y doce horas. Un $13 \%$ de los corresponsales asegura trabajar más de doce horas cada jornada. Si agregamos los dos datos anteriores se concluye que el $68 \%$ de los corresponsales, más de dos tercios de los mismos, trabaja de media más de diez horas al día. Hay incluso un $8 \%$ que trabaja más de quince horas al día.

Salvo contadas excepciones el corresponsal de radio está obligado a madrugar mucho. Esto se debe a que sus crónicas con información actualizada son necesarias y requeridas a primera hora de la mañana, que es la hora del prime time radiofónico y el momento del día en que las grandes cadenas de radio tienen un mayor número de oyentes. Como es lógico, antes de proceder a intervenir en los programas informativos matutinos, el corresponsal debe previamente haber hecho una lectura de la prensa, repasado los principales medios de comunicación locales y actualizado todos los contenidos.

La mayor parte de los corresponsales suele por ello levantarse muy temprano. Los datos obtenidos en el marco de esta investigación ponen de relieve que el $44 \%$ de los corresponsales, prácticamente la mitad de ellos, se levanta diariamente entre las cinco de la madrugada y las siete de la mañana. El $34 \%$, algo más de un tercio, sale de la cama y se pone a trabajar entre las siete y las ocho de la mañana. El $22 \%$ restante se levanta más tarde de las ocho. Esto suele ocurrir principalmente en las corresponsalias que se encuentran en otros husos horarios, sobre todo en las situadas en el este, como las de Oriente Medio y Asia.

El ciclo informativo de la corresponsalía de radio se prolonga durante todo el día. Si los programas informativos de la mañana son los que más audiencia tienen, le siguen en segundo lugar los de la noche, cuya audiencia es superior a la de los informativos de mediodía. Los programas informativos nocturnos suelen concluir a medianoche y eso, en ocasiones, exige que el corresponsal esté operativo hasta muy tarde.

En cualquier caso, aunque no tenga comprometido ningún directo en el programa informativo de la noche, el corresponsal está al tanto de las noticias hasta última hora. Ello le permite ver ya las portadas de la prensa del día siguiente o seguir los programas de las televisiones o radios locales en las que se realiza un análisis más reposado de lo que ha dado de sí la jornada. Casi la mitad de los corresponsales se acuesta pasada la medianoche. El $80 \%$ se va a la cama más tarde de las 23 horas.

La agenda del corresponsal está mucho menos cargada los fines de semana que los días de diario pero igual de expuesta a la ocurrencia de cualquier eventualidad. La tranquilidad del descanso puede verse alterada en el momento más inopinado. Una alerta en el teléfono móvil o una llamada desde la redacción central son susceptibles de romper cualquier jornada de asueto de sábado o domingo.

Sea como fuere, se produzcan o no noticias de alcance imprevistas, el corresponsal debe, todos los fines de semana, hacer un repaso, aunque sea somero, de la prensa local, atentos por ejemplo a las exclusivas de la prensa de los domingos, que suele ser con frecuencia objeto de noticia en países como los Estados Unidos, el Reino Unido, Francia o Alemania. Como resultado, la mayor parte de los corresponsales de radio acaba de una u otra manera trabajando los fines de semana. El $70 \%$ asegura que los trabajan «muchas veces» 0 «siempre». Ninguno ha respondido «nunca» 0 «casi nunca».

\section{Reflexiones de los corresponsales de radio sobre su futuro laboral}

En el marco de la presente investigación se les ha preguntado a los corresponsales de radio cómo ven el futuro de la figura del corresponsal de radio. A cada uno los 64 corresponsales en el extranjero de las cuatro grandes emisoras de radio generalistas se les ha asignado un número para identificarlos sin desvelar su nombre y apellidos y la emisora de radio para la que trabajan (C1, C2...C64).

C5 cree que el futuro de los corresponsales de radio es «similar al de los corresponsales de otros medios» y explica que seguirán siendo «necesarios pero precarizados». C43 ve el futuro del corresponsal de radio «tan mal como el del corresponsal de tele o el de prensa». C49 contempla la situación de forma «muy preocupante» y C26 describe el panorama que viene por delante como «muy negro».

En relación con las condiciones laborales, C29 cree que las cosas seguirán más o menos «como ahora porque les resulta cómodo», si bien pronostica que «los que tienen un sueldo fijo se convertirán en freelance». C9 opina que los corresponsales de radio seguirán «siendo necesarios para aportar su visión de una sociedad distinta, pero sus condiciones laborales serán cada vez más precarias". C21 dice:

«Mi visión es que en España se está precarizando la figura del corresponsal en radio. Cada vez son más escasos los corresponsales de plantilla. El futuro tiende a tener freelance a los que no se pagará por un fijo, sino por crónica enviada».

C14 cree que «si siguen tan pésimas las condiciones económicas, la figura del corresponsal será cada vez más exótica y solo la podrán desarrollar ricos de familia adictos a los medios, o supermujeres y superhombres que trabajen en varios medios al mismo tiempo». C44 augura más «precariedad» y constata que cada vez tiene más compañeros en su situación: «freelance con poco 
apoyo económico de la redacción». C17 vislumbra también un futuro aciago y no descarta la pronta desaparición de la figura del corresponsal fijo desplazado expresamente por la empresa a otro país para cubrir la información que allí se genere:

«Viendo como están las radios en España, lo veo mal. Y casi diría que serán prescindibles muy pronto en su formato de corresponsal fijo mandado desde Madrid. La SER, la COPE, Onda Cero, tienen solo colaboradores, a veces sin un contrato, y están siendo maltratados y mal pagados por sus empresas. Muchos tienen que buscarse otras fuentes de ingresos. Pero es el modelo que impera entre los españoles».

Hay profesionales como C33 que presagian que «el corresponsal desaparecerá por completo y solo se usarán periodistas freelance». C31 tiene claro que "los medios tienden a usar cada vez más al corresponsal freelance» y que el corresponsal tradicional está «en clara extinción porque la mayoría de los corresponsales al uso de radio han desaparecido fuera del eje Bruselas/Europa-Estados Unidos». En un sentido parecido, C59 pronostica que «la figura del corresponsal, exceptuando en las tres capitales mundiales, será la del freelance mal pagado sin ningún tipo de estabilidad que le permitiese hacer un mejor trabajo».

C2 realza la importancia de los corresponsales fijos y de tener un vínculo contractual con la empresa, y advierte de que el creciente uso de freelance por parte de las radios españolas puede, en su opinión, tener repercusiones en la calidad de la información producida:

«Existe el riesgo de que las radios prescindan de las figuras de corresponsales para depender de freelance. Ese riesgo tiene que ser medido ya que un free nunca sentirá la necesidad de competir por su empresa. La disponibilidad de alguien de plantilla puede ser exigida. Y por supuesto, la experiencia de mantener a alguien unos años en la corresponsalía te ofrece una visión temporal imposible de conseguir en quien acaba de aterrizar».

Para C59 «las radios españolas no apuestan por la información internacional y no les interesa porque es un gasto que no quieren asumir» y apunta también a una merma de la calidad de la información porque «mientras las radios sigan pagando como están pagando, recibirán desde las corresponsalías una calidad acorde a ese precio, y nada indica que vayan a pagar mejor».

De forma mayoritaria los corresponsales de las radios españolas creen que en el futuro los corresponsales no podrán hacer solo radio y deberán por lo tanto operar en otras plataformas como la prensa, la televisión o las agencias para poder sobrevivir. C54 describe la posibilidad de que un corresponsal pueda vivir en el futuro solo de la radio como una "utopía». "Vivir solo de esto no da dinero», asegura, "pero si lo complementas con otro trabajo lo veo factible y enriquecedor». C58 ve el futuro «demasiado precario y si es como freelance a la pieza -mi caso- casi imposible desarrollarlo como única fuente de ingresos». Por su parte, C7 opina que la situación no variará sustancialmente en el corto y medio plazo y predice que los corresponsales de radio seguirán siendo «profesionales multiplataforma, con capacidad de adaptación a los lenguajes informativos de distintos medios (radio, televisión, Internet), que puedan suplir la ausencia de una infraestructura sólida al estilo de las corresponsalías tradicionales».

Hay corresponsales que opinan que el corresponsal de radio del futuro no deberá únicamente operar en varias plataformas, sino que deberá también extender el radio de acción de sus corresponsalías para convertirlas en corresponsalías regionales. Es el caso de $\mathrm{C} 10$, que cree que «cambiará la figura pero el corresponsal en esencia seguirá existiendo aunque no será posible ni factible el corresponsal por país». En el futuro, explica, «los corresponsales deberán serlo por grandes áreas geográficas: Mediterráneo, África Central, Extremo Oriente... como ya se impone en algunas zonas como EEUU, Latinoamérica, países de habla germana u Oriente Próximo y tendrán que desplazarse a las diversas zonas de su competencia». C27 se rinde a la evidencia de que en el futuro los corresponsales serán «freelance o stringers pero es necesario que los periodistas estén especializados en su área y que las radios no tengan un corresponsal por tener». Pero para que el corresponsal puede desplazarse de forma habitual por una determinada región hacen falta medios económicos. En este sentido, C39 apunta lo siguiente:

«El futuro del corresponsal de radio es como el de tele, prensa escrita o medios digitales. No importa el medio, importa la historia que se cuenta, cómo se cuenta, quién la cuenta. El corresponsal de cualquier medio debe saber dar su mirada personal, propia, y contar con medios para moverse y llegar a las zonas de la noticia. Lo peor que le puede pasar a un corresponsal es que su medio le haga ser un quedado especial en vez de un enviado especial en la zona».

Con respecto a los contenidos y a la información cubierta por los corresponsales hay profesionales que están descontentos con el uso que las radios españolas hacen en la actualidad de sus corresponsales. C33 cree que en adelante «el trabajo que realizamos debería adaptarse a la realidad de los medios digitales y las redes sociales». Al contrario de lo ocurría en el pasado ahora hay varios medios que compiten con la radio para contar al instante lo que está pasando y por eso piensa que el "corresponsal debería no ir pegado a la inmediatez y sí tener tiempo para trabajar las historias y buscar fuentes propias». A C17 también le parece que se abusa del corresponsal a hora de producir piezas para diarios y boletines y se muestra partidario de reforzar el rol del corresponsal como analista e intérprete de la realidad del país en el que reside:

«Con este sistema de actuar y pensar de radio española, en el que lo que cuentan son los opinadores y tertulianos y en donde muy a menudo apenas se cuenta con el corresponsal que se encuentra en el lugar donde está produciéndose la noticia, es evidente que se ignora o vilipendia la experiencia y conocimientos de ese profesional. No es el caso de las radios públicas alemana y francesa, donde los corresponsales no entran en boletines por sistema, sino en diarios y con piezas de dos o tres minutos o en diálogos más pausados». 
C18 esgrime argumentos semejantes y apela a las radios españolas a que hagan un uso diferente del corresponsal:

«Creo que los medios deberían replantearse que los corresponsales no podemos ser simplemente agencias de información o loritos y que deberían dejarnos más tiempo para trabajar temas con profundidad para explicar bien qué ocurre en los países en los que vivimos. No obstante, nuestro trabajo entiendo que es un reflejo del tipo de periodismo que se hace en la madre patria, periodismo de declaraciones y lleno de lugares comunes y de simplezas».

La mayoría de los corresponsales de radio sienten que el encorsetamiento de las crónicas de cincuenta segundos no les permite explorar y analizar en toda su amplitud las claves de la actualidad en sus respectivos países de residencia. C16 reclama un papel más protagónico y más tiempo en antena para el corresponsal de radio.

«Me gustaría ver un futuro en el que el corresponsal dispusiera de más tiempo para poder aportar, no solo crónicas de urgencia, sino información más elaborada y con mayor grado de análisis. La experiencia y el conocimiento del corresponsal, por su presencia en el país y el contacto con su sociedad, han de poder quedar reflejados en su trabajo. Es el plus, el valor añadido que debiera esperarse de la figura del corresponsal, ya que los datos y la información básica hoy es accesible a todos gracias a las nuevas tecnologías».

Esas nuevas tecnologías a las que se refiere $\mathrm{C} 16$ confieren al mismo tiempo más movilidad a los corresponsales, que de un tiempo a esta parte disponen de más y mejores recursos para realizar conexiones desde lugares remotos, una tendencia que se va a agudizar en el futuro. $\mathrm{C} 7$ apuesta por que el corresponsal de radio salga más a la calle y aproveche las potencialidades que ofrecen las nuevas tecnologías para tener un contacto más directo con las fuentes y los protagonistas de las noticias.

«Creo que la figura del corresponsal es necesaria pero hay que dar un paso adelante. Creo que debemos salir más a la calle e intentar hacer radio fuera de casa. El gran error del corresponsal del siglo XXI es que tantas facilidades están minando la profesión por dentro. El periodismo y más la corresponsalía tiene que estar viva y en la calle».

De todo lo expuesto anteriormente se puede colegir que la figura del corresponsal de radio está siendo objeto de profundas transformaciones tanto en el plano laboral, como en el tecnológico o el de los contenidos. Las visiones sobre el futuro son por lo general negativas, y las perspectivas, poco halagüeñas. Aún así, consideran que los corresponsales de radio, cualesquiera que sean sus características definitorias, seguirán existiendo. C52 proclama que la figura del corresponsal de radio es «imprescindible», y C47 la califica de «necesaria». C18 asegura "ser optimista y quiero pensar que el corresponsal seguirá siendo importante para un medio y para el oyente contar con alguien de la casa para contar lo que ocurre en otros lugares». C13 cree que el corresponsal de radio seguirá siendo «un testigo inmediato y riguroso de la actualidad del país sobre el que informa y un profesional que explica y comprende por qué es interesante que la gente escuche lo que está contando». Para C11, el de radio, es quizá el corresponsal más imprescindible de todos:

«Posiblemente sea uno de los pocos corresponsales insustituibles. En la prensa escrita y la TV se tiende a reducir el número de corresponsalías y ampliar el de enviados especiales. En cambio, la radio necesita estar. No se puede improvisar la presencia. Creo, sinceramente, que el oyente nota absolutamente todo: si uno acaba de llegar a un sitio o ni siquiera está allí, las crónicas suenan a teletipo impersonal».

C25 es también optimista con respecto al futuro porque cree que «la radio tiene una magia especial, el poder escuchar a alguien que vio las cosas con sus propios ojos o que al menos está en el lugar en el que ocurrieron y puede transmitirlo fielmente; es insustituible». Con palabras similares describe la situación C35, para quien la figura del corresponsal de radio tiene «mucho futuro porque la radio es el medio más barato de todos los medios de comunicación, el que llega antes al público y el que le ofrece la posibilidad de mantenerse conectado siempre en cualquier lugar donde se encuentre». C57 está seguro de que el futuro está garantizado ya que «la radio (...) tiene un potencial muy grande y que los españoles, a pesar de los cambios culturales, siguen eligiendo la radio como compañera la mayor parte del día».

\section{Conclusiones}

Las presiones económicas han obligado a las grandes cadenas de radio españolas a reducir paulatinamente el número de corresponsales fijos que tienen en plantilla. Sin embargo, ha quedado también de manifiesto que las radios españolas necesitan y quieren disponer de profesionales del periodismo en distintos lugares del mundo para poder facilitar a sus audiencias información internacional de calidad sobre el terreno. El interés por tener corresponsales en el extranjero permanece y, de hecho, cada una de las cuatro cadenas generalistas dispone de más de una decena de informadores en diferentes países. Con la excepción de RNE, nunca han tenido las radios españolas a tantos periodistas informando desde el extranjero, algo que solo ha sido posible gracias a los avances que se han producido en el ámbito de las telecomunicaciones y de la informática, que están abaratando y facilitando la transmisión de información desde cualquier lugar del mundo.

A lo largo de los últimos años se ha producido una precarización de las condiciones laborales de los corresponsales de radio que ha llevado a muchos académicos a cuestionar incluso el futuro de los corresponsales en el extranjero. Pero más que desaparecer, la figura del corresponsal de radio se está transformando. De forma progresiva las radios sustituyen a los corresponsales fijos por corresponsales freelance; el corresponsal que antes se dedicaba en exclusiva a un único medio, presta 
cada vez más sus servicios a distintas empresas de comunicación; y el corresponsal que antaño trabajaba solo para la radio, está dando paso a un corresponsal versátil que opera en múltiples plataformas.

\section{Bibliografía}

Asociación de la Prensa de Madrid (2015). Informe Anual de la Profesión Periodística 2014. Madrid: APM.

Cea d'Ancona, M. A. (1998). Metodología cuantitativa. Estrategias y técnicas de investigación social. Editorial Síntesis.

Cohen, N. S. (2015). From Pink Slips to Pink Slime: Transforming Media Labor in a Digital Age. The Communication Review, 18(2), 98-122.

Enríquez, C. (2012) ¿Desaparecerán los corresponsales?. Periodistas, nº 30, FAPE.

Hamilton, J. M., y Jenner, E. (2004). Redefining foreign correspondence. Journalism, 5(3) 301-321.

Hannerz, U. (2012). Foreign news: Exploring the world of foreign correspondents. University of Chicago Press.

Hess, S. (1996). International news and foreign correspondents (Vol. 5). Brookings Institution Press.

Molina, M. (2012). ¿El corresponsal ha muerto? ¿Larga vida al corresponsal?. Periodistas, n³0, FAPE

Rábago, J. (2012). El corresponsal extranjero. Un oficio en extinción. La Nueva España. En http://www.Ine.es/opinion/2012/11/23/corresponsal-extranjero-oficio-extincion/1331023.html

Sahagún, F. (1986). El mundo fue noticia: corresponsales españoles en el extranjero: la información internacional en España. Madrid: Fundación Banco Exterior.

Sahagún, F. (2013). Corresponsales extranjeros, ¿especie en extinción o en transformación?. Cuadernos de periodistas: revista de la Asociación de la Prensa de Madrid, (21), 17-29.

Sambrook, R. (2010). Are foreign correspondents redundant?: the changing face of international news. Reuters Institute for the Study of Journalism, University of Oxford.

San Martín, E. (2012). Con nombres y apellidos. Periodistas, n³0, FAPE.

Sierra Bravo, R. (1994). Técnicas de investigación social: teoría y ejercicios. Paraninfo.

Terzis, G. (Ed) (2015). Mapping foreign correspondence in Europe. Routledge.

Tulloch, C. D. (1998). Los corresponsales en el extranjero de prensa diaria española y el proceso de comunicación de la información internacional. (Tesis Doctoral). Universitat Pompeu Fabra.

Tulloch, C. D. (2004). Corresponsales en el extranjero, mito y realidad. Ediciones Universidad de Navarra. EUNSA.

Vinuesa, M. L. (2005). La encuesta: Observación extensiva de la realidad social. Investigar en comunicación: guía práctica de métodos y técnicas de investigación social en comunicación, 177-206. McGraw-Hill Interamericana de España.

Williams, K. (2011) International Journalism. Sage.

Cómo citar este artículo en bibliografías - How to cite this article in bibliographies / references:

TATO, J. (2017): "El deterioro de las condiciones laborales de los corresponsales de radio". En Revista de la Asociación Española de Investigación de la Comunicación, vol. 4, número 7, pp. 157-165.

Notas

Esta investigación forma parte del proyecto Big data, redes sociales y periodismo de datos: aplicación de las herramientas de monitorización al análisis de fuentes y contenidos periodísticos, financiado por el Ministerio de Economía y Competitividad dentro del Plan Nacional Proyectos de I+D+I, del Programa Estatal de Investigación, Desarrollo e Innovación orientada a los Retos de la Sociedad. Referencia: CSO2013-47767-C2-1-R. Asimismo, se enmarca dentro de las acciones desarrolladas por el grupo de investigación Paseet de la Universidad Carlos III de Madrid. 